\title{
Metabolites in aging and autophagy
}

\author{
Sabrina Schroeder ${ }^{1, \#}$, Andreas Zimmermann ${ }^{1, \#}$, Didac Carmona-Gutierrez ${ }^{1}$, Tobias Eisenberg ${ }^{1}$, Christoph \\ Ruckenstuhl ${ }^{1}$, Aleksandra Andryushkova ${ }^{1}$, Tobias Pendl ${ }^{1}$, Alexandra Harger ${ }^{1,2}$ and Frank Madeo ${ }^{1, *}$ \\ ${ }^{1}$ Institute of Molecular Biosciences, University of Graz, Humboldtstrasse 50, 8010 Graz, Austria. \\ 2 Division of Endocrinology and Metabolism, Dept. of Internal Medicine, Medical University of Graz, Auenbruggerplatz 15, 8036 Graz, \\ Austria. \\ \# These authors contributed equally to this work. \\ * Corresponding Author: F. Madeo, Humboldtstrasse 50; 8010 Graz, Austria; Tel: +43 316380 8878; Fax: +43 316380 9898; E-mail: \\ frank.madeo@uni-graz.at
}

\begin{abstract}
INTRODUCTION
Autophagy, the main lysosomal degradative machinery, plays a major role in maintaining cellular homeostasis and thus a healthy state in an organism. This process recycles unnecessary or damaged material, therefore, not only providing nutrients to maintain vital cellular functions in times of starvation but also eliminating potentially harmful cellular material [1]. Importantly, the autophagic rate declines with increasing age [2,3], suggesting a functional correlation between aging and autophagy. Indeed, the deregulation of autophagy is involved in the onset of various age-related diseases such as cancer, cardiomyopathy, type II diabetes, and neurodegeneration [4]. Until recently, aging was regarded as an unregulated and inescapable consequence of the accumulation of incidental damage in macromolecules and/or organelles. However, the discovery of multiple ways to extend the lifespan in a variety of different model organisms, e.g., by genetic and pharmacological means, developed the formulation of alternative aging theories that consider aging as a molecular program [5]. Indeed, the last years have provided important insights into the networks that control aging and have thus highlighted the interconnected nature of aging and various cellular processes. For instance, the process of aging is intimately coupled to metabolic processes [6], in particular to energy metabolism and nutrient availability. Nevertheless, specific metabolites that affect aging and autophagy remain poorly described.
\end{abstract}

\section{NUTRIENT AVAILABILITY CONTROLS AUTOPHAGY AND AGING VIA ENERGY METABOLITES \\ As sensors of the current environmental status, nutrient signaling pathways represent central aging regulators. For instance, individual interventions in the insulin/insulin-like growth factor 1 (IGF-1), Ras, protein kinase A (PKA), target of rapamycin (Tor), or protein kinase $\mathrm{B}$ (SCH9/Akt) path- ways have been shown to extend lifespan in various organ-}

isms, including mammals [7-9]. Caloric restriction (CR) requires autophagy for lifespan extension [10-12] and CRmediated autophagy induction follows molecular pathways that are shared with those known to affect aging, such as Tor, SCH9/Akt, or IGF-1 [13]. Furthermore, the AMPactivated protein kinase (AMPK) serves as a metabolic radar sensing changes in the AMP/ATP ratio and is conserved in the majority of eukaryotic species, and has also been established as a checkpoint for growth control and autophagy regulation [14]. Consistently, several studies have revealed a connection between the AMP/ATP ratio, autophagic flux rates, senescence, and disease $[15,16]$. Noteworthy, early studies on rat hepatocytes also suggested that the execution of autophagy depends on energy availability since inhibition of ATP production stalls autophagic flux [17]. Other pivotal energy sources like butyrate, an essential energy component in the colon, and second messengers such as CAMP, which might also be implicated in Ras/PKA-mediated lifespan modulation in various organisms, were identified as potential autophagy mediators $[18,19]$. This argues for a decisive function of nutrient signaling and energy metabolites during aging and its associated processes.

\section{THE ENERGY METABOLITE ACETYL-COA SUPPRESSES} AUTOPHAGY VIA PROTEIN ACETYLATION

In the yeast $S$ cerevisiae, glucose is the preferred carbon source for fueling energy metabolism and its fermentation produces acetate and ethanol that is used for subsequent respiration after the diauxic shift. Both intermediates are released into the medium and have been attributed a role in limiting yeast chronological life span [20], but considered a pro-aging factor specific for yeast [21]. However, acetate is an important metabolite that is involved in central processes such as acetyl-Coenzyme A (acetyl-CoA) production. Thus, its metabolic fate rather than a simply extracellular toxicity may (at least in part) be responsible for its impact 
on lifespan. Consequently, acetate metabolism may also contribute to aging in higher eukaryotes, possibly via its impact on protein acetylation through acetyl-CoA generation and subsequent control of cellular function. Energy metabolites that can derive in the production of acetyl-CoA, such as citrate, pyruvate and fatty acids, were shown to be deregulated in senescence-accelerated mice [22]. We could recently show that (nucleo-)cytosolic acetyl-CoA, in fact, serves as a modulator of longevity, suppressing starvation and age-associated autophagy in a variety of phyla $[23,24]$. This function might be due to the fact that acetyl$\mathrm{COA}$ is the only donor for acetylation reactions and both, protein acetylation and epigenetic chromatin modifications, have repeatedly been linked to the regulation of aging and autophagy $[23,25,26]$. For instance, the highly conserved protein family of NAD+-dependent histone deacetylases and ADP ribosylases (sirtuins) has been connected to aging modulation [27,28] and lifespan extension upon CR [29]. Activation of histone deacetylases, such as sirtuins, has therefore been extensively studied for its capability to combat aging or age-associated pathologies. Interestingly, the lifespan-extending effects of sirtuin activation by CR or pharmacological interventions depend on the induction of autophagy [11]. The dependency on NAD+ as a cofactor

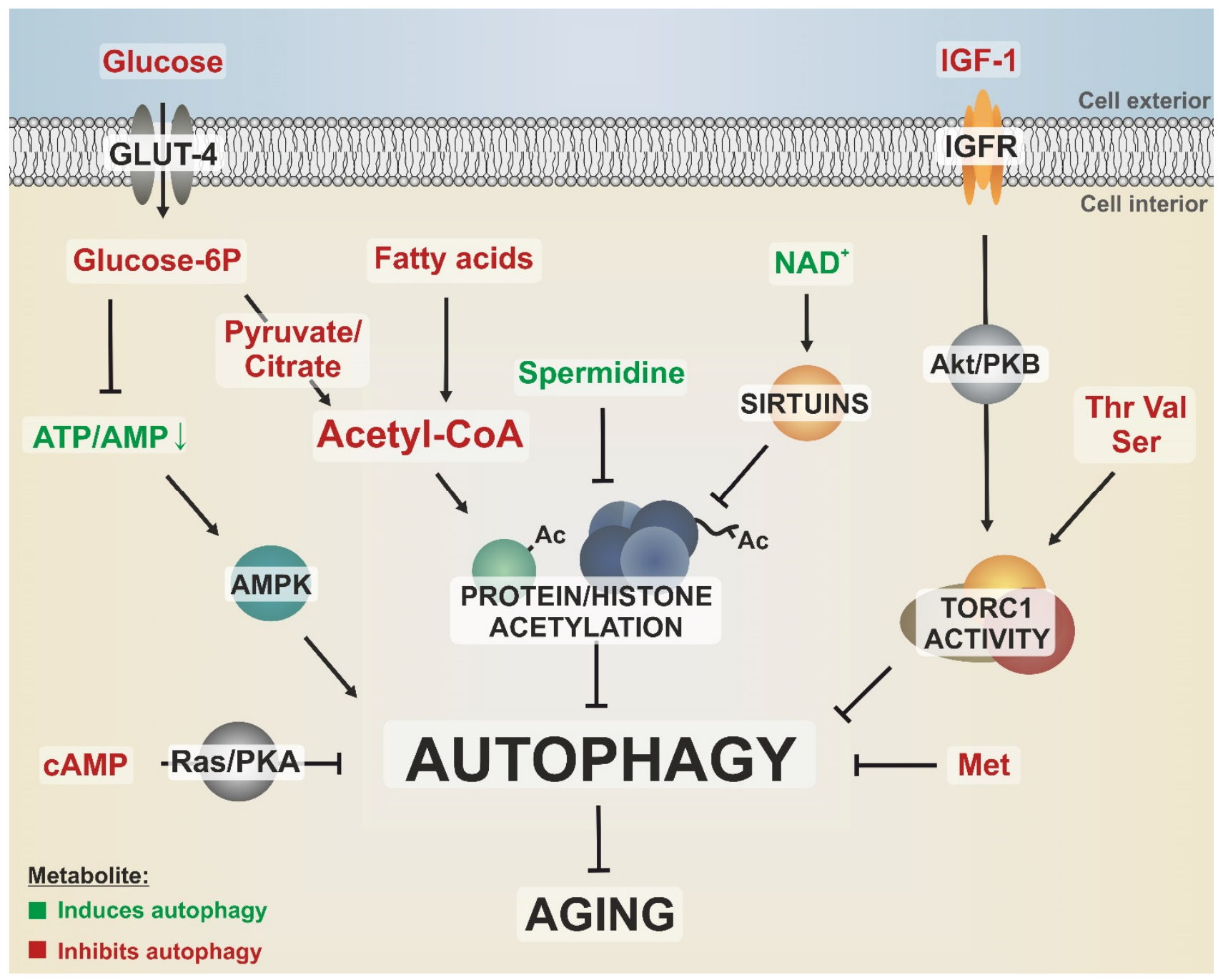

FIGURE 1: Different metabolites converge on pathways that regulate autophagy and aging. Dietary nutrients like glucose, amino acids and fatty acids as well as growth signaling by IGF-1 activate nutrient-sensing kinases, like the target of rapamycin complex 1 (TORC1), which stalls autophagy via phosphorylation of downstream targets. Furthermore, autophagy is negatively regulated by the Ras/PKA pathway, which responds to nutrient availability by sensing intracellular cAMP levels. The cellular energy status is reflected by the ATP/AMP ratio, which is sensed by the autophagy activator AMPK. Methionine downregulates autophagy during aging in a yet to be elucidated fashion. The central energy intermediate acetyl-CoA integrates metabolites from glycolysis, $\beta$-oxidation or respiration and fuels acetylation of proteins such as histones, resulting in decreased autophagic flux. All these autophagy-limiting metabolic pathways have been linked to an accelerated aging phenotype. In contrast, polyamines, like spermidine, reduce protein acetylation, thereby promoting autophagy and longevity. Potential crosstalks between protein acetylation and nutrient sensing kinase signaling are yet to be elucidated. GLUT-4, glucose transporter 4; IGFR, IGF-1 receptor; Ac, acetyl-group; amino acids are indicated by three-letter code. 
and the tight connections between sirtuins, longevity and autophagy induction have led to the hypothesis that sirtuins act as metabolic sensors that promote mitochondrial maintenance [30]. Notably, nicotinamide metabolism has become an intensively investigated target for drug discovery against a variety of human diseases, including age-associated pathologies such as cancer or neurodegeneration [31].

\section{POLYAMINE METABOLISM LEAVES A REGULATORY FINGERPRINT AT HISTONE MODIFICATION SITES}

The levels of polyamines, a class of ubiquitously occurring small basic polycations, decline with progressing age in various organisms, including humans [32], yeast [25] and plants [33]. Intriguingly, external application of a specific polyamine (spermidine) counteracts cell death during aging and improves the lifespan of yeast, flies, worms, and human immune cells in an autophagy-dependent manner [25] Furthermore, it causes a reduction of oxidative stress in mice. In addition, more recent studies also suggest longevity-promoting effects in mammals [34]. Importantly, spermidine treatment appears to be associated with both histone hypoacetylation caused by inhibition of histone acetyl transferases [25] and deacetylation of cytosolic proteins [35]. However, it has also been hypothesized that polyamines influence histone acetylation in dependence of the histones' own acetylation status [36] and massive polyamine catabolism has even been shown to deplete the acetylation co-factor acetyl-CoA [37]. Possibly, the involvement of spermidine in the biosynthesis of the methyl-group donor S-adenosyl-methionine (SAM) [38] could also culminate in regulatory methylation reactions, such as chromatin silencing. Thus, polyamines may influence chromatin structure and protein acetylation via multiple mechanisms. Given the aforementioned contribution of acetyl-CoA to aging and autophagy modulation, it is tempting to speculate that spermidine functions include a down-titration of the intracellular acetyl-CoA pool and thus a rearrangement of the metabolic state.

\section{SPECIFIC AMINO ACID STARVATION INDUCES AUTOPHAGY AND PROLONGS LIFESPAN}

In line with the vast importance of nutrient signaling during aging, amino acid metabolism has an important impact on eukaryotic aging and its related diseases. The levels of specific amino acids like tryptophane, methionine, arginine, or leucine has often been suggested to (positively or negatively) influence the autophagy pathway and impact aging in different eukaryotes [39-42]. Indeed, we could recently demonstrate that limitation of the amino acid methionine enhances yeast chronological lifespan. Intriguingly, this lifespan extension requires autophagy-dependent vacuolar acidification [43]. In the same line, vacuolar acidification also elongates replicative lifespan of yeast, where it protects mitochondria most probably via an improvement of the vacuolar amino acid storage function [44]. In yeast, serine, threonine and valine promote cellular senescence probably via activation of the Sch9/TOR pathway and subsequent inhibition of the protein kinase Rim15p, which guides anti-aging stress response pathways [45]. Altogether, these examples suggest that the intake or limitation of specific amino acids is a determining factor during aging, though the mechanistic specificities are expected to be complexly regulated, especially at the organismal level.

\section{CONCLUSION}

A variety of potential metabolic controllers of autophagy and health span have already been proposed. However, precise strategies to target the correlating pathways (e.g., by nutrition patterns) remain to be elucidated in more detail. For example, it would be of great interest to determine if special diets that include the limitation of (defined) amino acids or the uptake of certain polyamines, like spermidine, influence the metabolism towards improved cellular conditions during aging. It also remains elusive how certain diets may affect the microbiome and in turn impact the levels of certain metabolites that have been shown to regulate cellular fitness, such as citrate, pyruvate, butyrate, or acetate. The investigation of metabolites as powerful rheostats in aging and autophagy is supported by the improvement of technologies that have opened up new possibilities to detect and trace even small molecules in vitro and in vivo. This might bring up metabolomics as a future trend for aging analyses [46]. Hopefully, the findings on the impact of metabolism on aging will culminate in amended dietary guidelines that would make eating the tastiest of all medicines.

\section{CONFLICT OF INTEREST}

The authors declare no conflict of interest.

\section{COPYRIGHT}

(C) 2014 Schroeder et al. This is an open-access article released under the terms of the Creative Commons Attribution (CC BY) license, which allows the unrestricted use, distribution, and reproduction in any medium, provided the original author and source are acknowledged.

Please cite this article as: Sabrina Schroeder, Andreas Zimmermann, Didac Carmona-Gutierrez, Tobias Eisenberg, Christoph Ruckenstuhl, Aleksandra Andryushkova, Tobias Pendl, Alexandra Harger and Frank Madeo (2014). Metabolites in aging and autophagy. Microbial Cell 1(4): 110-114. doi: 10.15698/mic2014.04.142 


\section{REFERENCES}

1. Kroemer G, Mariño G, and Levine B (2010). Autophagy and the integrated stress response. Mol Cell 40(2): 280-293.

2. Cuervo AM (2008). Autophagy and aging. Trends Genet TIG 24(12): 604-612.

3. Simonsen $A$, Cumming RC, Brech $A$, Isakson $P$, Schubert DR, Finley KD, Simonsen A, Cumming RC, Brech A, Isakson P, and Finley DRS and KD (n.d.). Promoting basal levels of autophagy in the nervous system enhances longevity and oxidant resistance in adult Drosophila. Autophagy 4(2): 176-184.

4. Levine B and Kroemer G (2008). Autophagy in the pathogenesis of disease. Cell 132(1): 27-42.

5. Goldsmith TC (2012). On the programmed/non-programmed aging controversy. Biochem Mosc 77(7): 729-732.

6. Houtkooper RH, Williams RW, and Auwerx J (2010). Metabolic networks of longevity. Cell 142(1).

7. Longo VD (1999). Mutations in signal transduction proteins increase stress resistance and longevity in yeast, nematodes, fruit flies, and mammalian neuronal cells. Neurobiol Aging 20(5): 479-486.

8. Tatar M, Kopelman A, Epstein D, Tu M-P, Yin C-M, and Garofalo RS (2001). A Mutant Drosophila Insulin Receptor Homolog That Extends Life-Span and Impairs Neuroendocrine Function. Science 292(5514): 107-110.

9. Kaeberlein M, Powers RW 3rd, Steffen KK, Westman EA, Hu D, Dang N, Kerr EO, Kirkland KT, Fields S, and Kennedy BK (2005). Regulation of yeast replicative life span by TOR and Sch9 in response to nutrients. Science 310(5751): 1193-1196.

10. Jia K and Levine B (2007). Autophagy is required for dietary restriction-mediated life span extension in C. elegans. Autophagy 3(6): 597-599.

11. Morselli E, Maiuri MC, Markaki M, Megalou E, Pasparaki A, Palikaras K, Criollo A, Galluzzi L, Malik SA, Vitale I, Michaud M, Madeo F, Tavernarakis N, and Kroemer $\mathrm{G}$ (2010). Caloric restriction and resveratrol promote longevity through the Sirtuin-1-dependent induction of autophagy. Cell Death Dis 1: e10.

12. Minina EA, Sanchez-Vera V, Moschou PN, Suarez MF, Sundberg E, Weih $M$, and Bozhkov PV (2013). Autophagy mediates caloric restriction-induced lifespan extension in Arabidopsis. Aging Cell 12(2): 327-329.

13. Rubinsztein DC, Mariño G, and Kroemer G (2011). Autophagy and Aging. Cell 146(5): 682-695.

14. Mihaylova MM and Shaw RJ (2011). The AMP-activated protein kinase (AMPK) signaling pathway coordinates cell growth, autophagy, \& metabolism. Nat Cell Biol 13(9): 1016-1023.

15. Yang Z, Fujii H, Mohan SV, Goronzy JJ, and Weyand CM (2013). Phosphofructokinase deficiency impairs ATP generation, autophagy, and redox balance in rheumatoid arthritis T cells. J Exp Med 210(10): 2119-2134.

16. Kubli DA and Gustafsson ÅB (2014). Cardiomyocyte health: adapting to metabolic changes through autophagy. Trends Endocrinol Metab 25(3): 156-164.

17. Plomp PJAM, Wolvetang EJ, Groen AK, Meijer AJ, Gordon PB, and Seglen PO (1987). Energy dependence of autophagic protein degradation in isolated rat hepatocytes. Eur J Biochem 164(1): 197-203.

18. Holen I, Gordon PB, Strømhaug PE, and Seglen PO (1996). Role of CAMP in the regulation of hepatocytic autophagy. Eur J Biochem FEBS 236(1): 163-170.
19. Donohoe DR, Garge N, Zhang X, Sun W, O'Connell TM, Bunger MK, and Bultman SJ (2011). The Microbiome and Butyrate Regulate Energy Metabolism and Autophagy in the Mammalian Colon. Cell Metab 13(5): 517-526.

20. Mirisola MG and Longo VD (2012). Acetic acid and acidification accelerate chronological and replicative aging in yeast. Cell Cycle 11(19): 3532-3533.

21. Burhans WC and Weinberger M (2009). Acetic acid effects on aging in budding yeast: Are they relevant to aging in higher eukaryotes? Cell Cycle 8(14): 2300-2302.

22. Jiang $N$, Yan $X$, Zhou $W$, Zhang $Q$, Chen $H$, Zhang $Y$, and Zhang $X$ (2008). NMR-Based Metabonomic Investigations into the Metabolic Profile of the Senescence-Accelerated Mouse. J Proteome Res 7(9): 3678-3686.

23. Eisenberg $T$, Schroeder $S$, Andryushkova A, Pendl $T$, Küttner $V$, Bhukel A, Mariño G, Pietrocola F, Harger A, Zimmermann A, Moustafa $T$, Sprenger A, Jany E, Büttner S, Carmona-Gutierrez D, Ruckenstuhl C, Ring J, Reichelt W, Schimmel K, Leeb T, Moser C, Schatz S, Kamolz L-P, Magnes C, Sinner F, Sedej S, Fröhlich K-U, Juhasz G, Pieber TR, Dengjel J, Sigrist SJ, Kroemer G, and Madeo F (2014). Nucleocytosolic Depletion of the Energy Metabolite Acetyl-Coenzyme A Stimulates Autophagy and Prolongs Lifespan. Cell Metab 19(3): 431-444.

24. Mariño G, Pietrocola $F$, Eisenberg $T$, Kong $Y$, Malik SA, Andryushkova A, Schroeder S, Pendl T, Harger A, Niso-Santano M, Zamzami N, Scoazec M, Durand S, Enot DP, Fernández ÁF, Martins I, Kepp O, Senovilla L, Bauvy C, Morselli E, Vacchelli E, Bennetzen M, Magnes C, Sinner F, Pieber T, López-Otín C, Maiuri MC, Codogno $P$, Andersen JS, Hill JA, Madeo F, and Kroemer G (n.d.). Regulation of Autophagy by Cytosolic Acetyl-Coenzyme A. Mol Cell 53(5): 710-725.

25. Eisenberg $T$, Knauer $H$, Schauer A, Büttner S, Ruckenstuhl C, Carmona-Gutierrez D, Ring J, Schroeder S, Magnes C, Antonacci L, Fussi H, Deszcz L, Hartl R, Schraml E, Criollo A, Megalou E, Weiskopf D, Laun P, Heeren G, Breitenbach M, Grubeck-Loebenstein B, Herker E, Fahrenkrog B, Fröhlich K-U, Sinner F, Tavernarakis N, Minois N, Kroemer $G$, and Madeo F (2009). Induction of autophagy by spermidine promotes longevity. Nat Cell Biol 11(11): 1305-1314.

26. Imai S, Armstrong CM, Kaeberlein M, and Guarente L (2000). Transcriptional silencing and longevity protein Sir2 is an NAD-dependent histone deacetylase. Nature 403(6771): 795-800.

27. Fabrizio P, Gattazzo C, Battistella L, Wei M, Cheng C, McGrew K, and Longo VD (2005). Sir2 blocks extreme life-span extension. Cell 123(4): 655-667.

28. Kaeberlein M, McVey $M$, and Guarente L (1999). The SIR2/3/4 complex and SIR2 alone promote longevity in Saccharomyces cerevisiae by two different mechanisms. Genes Dev 13(19): 2570-2580.

29. Lin S-J, Defossez P-A, and Guarente L (2000). Requirement of NAD and SIR2 for Life-Span Extension by Calorie Restriction in Saccharomyces cerevisiae. Science 289(5487): 2126-2128.

30. Sack MN and Finkel T (2012). Mitochondrial Metabolism, Sirtuins, and Aging. Cold Spring Harb Perspect Biol 4(12): a013102.

31. Khan JA, Forouhar F, Tao X, and Tong L (2007). Nicotinamide adenine dinucleotide metabolism as an attractive target for drug discovery. Expert Opin Ther Targets 11(5): 695-705.

32. Scalabrino $G$ and Ferioli ME (1984). Polyamines in mammalian ageing: an oncological problem, too? A review. Mech Ageing Dev 26(2-3): 149-164. 
33. Kaur-Sawhney R, Shih L-M, Flores HE, and Galston AW (1982). Relation of Polyamine Synthesis and Titer to Aging and Senescence in Oat Leaves 1. Plant Physiol 69(2): 405-410.

34. Soda K, Dobashi Y, Kano Y, Tsujinaka S, and Konishi F (2009). Polyamine-rich food decreases age-associated pathology and mortality in aged mice. Exp Gerontol 44(11): 727-732.

35. Morselli E, Marino G, Bennetzen MV, Eisenberg T, Megalou E, Schroeder S, Cabrera S, Benit P, Rustin P, Criollo A, Kepp O, Galluzzi L, Shen S, Malik SA, Maiuri MC, Horio Y, Lopez-Otin C, Andersen JS, Tavernarakis $N$, Madeo $F$, and Kroemer $G$ (2011). Spermidine and resveratrol induce autophagy by distinct pathways converging on the acetylproteome. J Cell Biol 192(4): 615-629.

36. Liu B, Sutton A, and Sternglanz R (2005). A Yeast Polyamine Acetyltransferase. J Biol Chem 280(17): 16659-16664.

37. Kee K, Foster BA, Merali S, Kramer DL, Hensen ML, Diegelman $\mathrm{P}$, Kisiel N, Vujcic S, Mazurchuk RV, and Porter CW (2004). Activated polyamine catabolism depletes acetyl-CoA pools and suppresses prostate tumor growth in TRAMP mice. J Biol Chem 279(38): 40076-40083.

38. Sauter M, Moffatt B, Saechao MC, Hell R, and Wirtz M (2013). Methionine salvage and S-adenosylmethionine: essential links between sulfur, ethylene and polyamine biosynthesis. Biochem J 451(2): 145-154.

39. Dillon EL (2013). Nutritionally essential amino acids and metabolic signaling in aging. Amino Acids 45(3): 431-441.

40. Orentreich N, Matias JR, DeFelice A, and Zimmerman JA (1993). Low Methionine Ingestion by Rats Extends Life Span. J Nutr 123(2): 269-274.
41. Van der Goot AT and Nollen EAA (2013). Tryptophan metabolism: entering the field of aging and age-related pathologies. Trends Mol Med 19(6): 336-344.

42. Aris JP, Alvers AL, Ferraiuolo RA, Fishwick LK, Hanvivatpong A, Hu D, Kirlew C, Leonard MT, Losin KJ, Marraffini M, Seo AY, Swanberg V, Westcott JL, Wood MS, Leeuwenburgh C, and Dunn Jr. WA (2013). Autophagy and leucine promote chronological longevity and respiration proficiency during calorie restriction in yeast. Exp Gerontol 48(10): 1107-1119.

43. Ruckenstuhl C, Netzberger C, Entfellner I, Carmona-Gutiérrez D, Kickenweiz T, Stekovic S, Gleixner C, Smidt C, Klug L, Sorgo AG, Eisenberg T, Büttner S, Marino $G$, Rafal K, Jansen-Dürr P, Fröhlich K-U, Kroemer $G$, and Madeo $F$ (2014). Lifespan extension by methionine restriction requires autophagy-dependent vacuolar acidification: in press.

44. Hughes $A L$ and Gottschling DE (2012). An early age increase in vacuolar $\mathrm{pH}$ limits mitochondrial function and lifespan in yeast. Nature 492(7428): 261-265.

45. Mirisola MG, Taormina G, Fabrizio P, Wei M, Hu J, and Longo VD (2014). Serine- and Threonine/Valine-Dependent Activation of PDK and Tor Orthologs Converge on Sch9 to Promote Aging. PLoS Genet 10(2): e1004113.

46. Kristal BS and Shurubor YI (2005). Metabolomics: Opening Another Window into Aging. Sci Aging Knowl Environ 2005(26): pe19. 